\title{
Anatomía de las Arterias de la Cabeza: Un Recurso Educativo Abierto como Herramienta Digital de Apoyo a la Enseñanza de la Anatomía
}

\author{
Anatomy of the Arteries of the Head: An Open Educational Resource \\ as a Digital Tool to Support the Teaching of Anatomy
}

Sandra Bucarey Arriagada*; Pedro Aravena Torres*; Ilse Pradenas Mera** \& Gonzalo Tiznado Matzner*

BUCAREY, A. S.; ARAVENA, T. P.; PRADENAS, M. I.; TIZNADO, M. G. Anatomía de las arterias de la cabeza: un recurso educativo abierto como herramienta digital de apoyo a la enseñanza de la anatomía. Int. J. Morphol., 34(4):1285-1292, 2016.

RESUMEN: El estudio de la anatomía, de acuerdo a anatomistas y estudiantes alrededor del mundo, requiere del trabajo práctico frente al cadáver y, por reducción de tiempo en la enseñanza de esta disciplina, que estas etapas sean fuertemente apoyadas por tecnologías educativas para responder a las demandas de una sociedad globalizada que promueve la equidad de la educación. En este contexto, una buena respuesta son los "Recursos Educativos Abiertos" (REA). El movimiento de los REA ha tenido un éxito significativo al promover la idea de que el conocimiento es un bien universal. Desde sus comienzos, los REA se consolidaron como claves para la democratización del conocimiento, dado que están disponibles en Internet, con licencia Creative Commons. A través de la Iniciativa Latinoamericana de Libros de Texto abiertos: Proyecto LATIn, se construyó un libro digital para estudiantes del área de la salud llamado Anatomía de las Arterias de la Cabeza, libro que se organizó de tal modo de integrar adecuadamente los contenidos desde la perspectiva pedagógica como multimedial. El libro cumple con las características de un REA, dado que está disponible y fácilmente accesible en Internet, además a través del Proyecto LATin está debidamente difundido en las 12 universidades que conforman la red del proyecto. Este libro se utilizó en la unidad de Sistema Circulatorio, en la asignatura de Anatomía Humana (ANAT 122) que se dictó a estudiantes de la Escuela de Obstetricia y Puericultura el primer semestre del año 2014 y del primer semestre 2015. El libro fue evaluado por un total de 101 estudiantes con un estudio descriptivo para determinar la satisfacción desde la perspectiva pedagógica y tecnológica. Los resultados arrojaron una alta satisfacción respecto del recurso, fluctuando entre el 76,4 \% y $100 \%$ de satisfacción en los diversos ítems.

PALABRAS CLAVE: Anatomía; Enseñanza; Recursos educativos abiertos.

\section{INTRODUCCIÓN}

Enseñanza de la Anatomía Humana para las nuevas generaciones

Tradicionalmente, la enseñanza de la anatomía humana requiere un importante número de horas frente al material cadavérico, con actividades prácticas que van desde la disección a la observación y reconocimiento de las diversas estructuras que conforman órganos y piezas cadavéricas. Estos pasos requieren que el estudiante logre orientación espacial, es decir, se ubique por diversos planos, cortes, alturas y niveles de profundidad para reconocer lo que estudia. En general, los estudiantes valoran y creen que es la enseñanza con el cadáver como más aprenden (Kurkcuoglu et al., 2015). Por otra parte, la poca cantidad de cadáveres, la cantidad de material correctamente diseccionado y la cada vez mayor cantidad de estudiantes por asignatura, dificultan una buena práctica del estudio de la anatomía en el pabellón. Al respecto, Inzunza (2014) hace referencia a la creciente dificultad que tienen algunos centros universitarios en Chile para acceder a material cadavérico.

Por otro lado, diversos autores han informado que en lo referente a las ciencias de la salud, las instituciones superiores en todo el mundo han estado experimentando nuevas modalidades de aprendizaje, las cuales, en general redundan en un descenso de horas y contenidos para la enseñanza de la anatomía (Nayak et al., 2006; Inzunza; Bergman et al., 2008; Fitzgerald et al., 2008; Patel \& Moxham, 2006) aspecto que inquieta a académicos, médicos en ejercicio y en formación: El tiempo dedicado a estu-

* Universidad Austral de Chile, Valdivia, Chile.

** Universidad de Antofagasta, Antofagasta, Chile.

Financiamiento: LATIn Project, Programa Alfa de la Unión Europea. 
diar la anatomía ha disminuido en medio de controversias de cómo, qué y cuándo son mejor entregados los contenidos de esta disciplina. Este reduccionismo, por ejemplo, ha provocado preocupación entre los facultativos, debido a que las nuevas generaciones de sus colegas egresan de la escuela de medicina con conocimientos anatómicos insuficientes. Sumado a que los nuevos médicos que inician el ejercicio de la profesión, en general, perciben la necesidad de un mayor énfasis en la enseñanza de la anatomía (Nayak et al.; Fitzgerald et al.; Waterston \& Steward, 2005; Kurkcuoglu et al.).

Un ejemplo de lo señalado anteriormente es el caso del Aprendizaje Basado en Problemas (ABP), en el cual Nayak et al., señalan que esta modalidad no cubre todos los objetivos de aprendizaje y que la anatomía aprendida a través de esta metodología es deficiente porque los estudiantes terminan priorizando los objetivos de otras disciplinas. Esto mismo es percibido por parte de los académicos, respecto a las mallas de bloques integrados, como es el caso de la Escuela de Medicina de la Universidad Austral, donde contenidos fundamentales de la anatomía como los sistemas: cardiovascular, musculo esquelético y nervioso, se ven afectados por la priorización de objetivos de otras disciplinas.

Patel \& Moxham aseguran que en un estudio realizado en institutos de educación superior, el $69 \%$ de los anatomistas está a favor de la utilización de la disección de cadáveres humanos sobre otros métodos de enseñanza, debido a que aparentemente permite lograr una gama de objetivos en diferentes cursos. De un listado de preferencias que se les presentó, los académicos priorizaron, como mejor método para la enseñanza, las lecciones prácticas que utilizan la disección de cadáveres por parte de los estudiantes. Así mismo, en otro estudio, Kurkcuoglu et al., sostienen que el $98 \%$ de los anatomistas profesionales creen que la anatomía macroscópica tiene un papel importante que jugar en la medicina clínica.

Frente a la revisión literaria que reafirma la importancia de usar el material cadavérico en la enseñanza de la anatomía y sumado a la escases de material en los laboratorios, situación general en los institutos y departamentos de anatomía humana, se cree que una manera de fortalecer la docencia en tiempos más acotados, es respaldando energicamente las nuevas tecnologías.

La realidad es, que no es posible volver hacia atrás con la cantidad de horas que se dedicaban antiguamente a la enseñanza de esta disciplina. Muchas universidades en el mundo, aprovechando los avances y cada vez mayor accesibilidad de tecnologías educativas, han propuesto nuevos métodos para enriquecer y aprovechar al máximo posible el tiempo disponible para la docencia. Un claro ejemplo es lo llevado a cabo por la Pontificia Universidad Católica de Chile, que para apoyar y fortalecer el trabajo práctico con cadáveres, dispuso de un variado número de plastinados y preparaciones anatómicas propias, que fotografiaron y luego digitalizaron como animaciones de tipo Flash, creando así módulos de auto-instrucción. A través de estudios pedagógicos estadísticos, demostraron el gran impacto que producían en el aprendizaje de los alumnos, lo cual se tradujo en un aumento estadísticamente significativo de las notas obtenidas en las evaluaciones prácticas (Inzunza \& Bravo, 2002; Bravo, 2006).

\section{Recursos Educativos Abiertos: posibilidad para la de- mocratización de la enseñanza de la anatomía humana}

La flexibilización de las estructuras académicas apuntan a un enfoque interdisciplinar y transdisciplinar en la concepción de nuevos currículos: la introducción de métodos activos y por otro lado el surgimiento de las Tecnologías de las Comunicaciones (TIC). Todos estos factores hicieron posible, como en muchos ámbitos, la globalización en la educación y como consecuencia de ello, la caída de las barreras para la universalización de ésta (Arboleda \& Rama, 2013).

Un movimiento de cambios por la masificación del acceso a Internet a partir de 1994, cuya incorporación a los procesos educacionales ha instalado un debate en torno a aspectos importantes subyacentes a la dinámica del proceso de enseñanza-aprendizaje con vistas a una nueva era: papeles, tiempo, espacio, saberes, etc. Todos ellos pasan a ser vistos a partir de la óptica de la construcción colaborativa y de la socialización del conocimiento, posibilitando la creación de nuevos contextos de innovación, donde colaboración, cooperación, autogestión, autonomía e intercambio de papeles son viables, superando de ese modo esquemas tradicionales. (Bucarey et al., 2013).

El término Recursos Educativos Abiertos (REA) fue establecido en el año 2005, durante el Foro sobre el Impacto de Materiales de Cursos Abiertos de la UNESCO (Universidad Virtual-REUNA, 2003), teniendo como base la gran impresión alcanzada por el movimiento del Software de Código Abierto. Este término fue acuñado para describir la provisión de recursos educativos de licencia abierta, posibilitada por las tecnologías de la información y la comunicación, para la consulta, uso y adaptación por parte de una comunidad de usuarios sin fines comerciales (Inamorato et al., 2012).

El movimiento de los REA ha tenido un éxito significativo al promover la idea de que el conocimiento es un bien universal, con un gran número de iniciativas en desarrollo (Ehlers, 2011). Desde sus comienzos estos recursos 
se consolidaron como claves para la democratización del conocimiento, conquistando el apoyo de instituciones, gobiernos e individuos de todo el mundo y han demostrado ser herramientas beneficiosas para la difusión del conocimiento con énfasis en la distribución y reutilización legal de los recursos. Algunos de estos beneficios son: el aprendizaje informal bajo demanda, la capacitación profesional abierta y continua, el acceso a nuevas prácticas pedagógicas, el estímulo del aprendizaje continuado y la colaboración entre pares para la mejoría de la calidad de los recursos producidos, con el consecuente aumento de la visibilidad de las instituciones protagonistas de este movimiento.

De acuerdo con Inamorato $e t$ al., a diferencia de aquellos contenidos genéricos abiertos que pueden ser encontrados en la web, los REA ponen énfasis en la distribución y reutilización legal de los recursos, con el uso de licencias abiertas, por ejemplo, Creative Commons, y la implementación de mecanismos para la evaluación de su calidad.

Los REA ganaron un impulso decisivo para su consolidación a través de iniciativas pioneras tales como el OpenCourseWare, del MIT, el OpenLearn, de la Open University del Reino Unido y Connexions, de la Rice University de los Estados Unidos, las que se unieron en una serie de nuevas iniciativas con el objetivo de implementar repositorios para distribución a través de la web, además de estimular la discusión y la creación colaborativa de prácticas de reutilización, metodologías de investigación y criterios de calidad.

Desde una definición más pedagógica, un Recurso Educativo Abierto se interpreta como una entidad informativa digital desarrollada para la generación de conocimiento, habilidades y actitudes, que tiene sentido en función de las necesidades del sujeto y que corresponde con una realidad concreta (Ramírez, 2007).

La anatomía humana, como se discutió anteriormente, no escapa a la necesidad de innovar en su enseñanza y de buscar utilizar de mejor forma las nuevas herramientas tecnológicas disponibles y de esa manera, apoyar y subsanar fuertemente el cada vez menor tiempo de estudio frente al material cadavérico. Así como, responder a las necesidades de la universalización de la enseñanza, del acceso equitativo a la formación de nuevas generaciones de estudiantes cada vez más permeados con el uso de las TIC.

\section{El proyecto LATin}

Una experiencia reciente de Recursos Educativos Abiertos para la Universidad Austral de Chile, ha sido la
Iniciativa Latinoamericana de Libros de Texto abiertos (LATIn), proyecto financiado por la Unión Europea en el marco de su Programa ALFA III EuropeAid (http:// www.proyectolatin.org/index.php/en/). El Proyecto LATIn está conformado por 12 universidades representantes de países de Centro y Sur América. El objetivo de este proyecto fue permitir el acceso a estudiantes de habla hispana que no siempre cuentan con los recursos para comprar libros especializados de alto costo y además con traducciones que no siempre son exactas. En esta propuesta se ha generado un libro de contenidos anatómicos en la Facultad de Medicina llamado Anatomía de las Arterias de la Cabeza, el cual se puede descargar desde la página web de LATin (http:// www.proyectolatin.org/index.php/en/library). Lo interesante de este recurso, es que además de hacerse accesible por web, se puede trabajar la integración de sus contenidos de manera innovadora para esta área del conocimiento al usar diversos recursos multimediales. En el área de la salud en general, los textos generados en habla hispana son escasos, la mayor parte son traducciones, a veces con bastantes errores y no siempre con una adecuada integración imagen-texto, aspecto fundamental para la mayor comprensión en el caso de la anatomía.

Se considera importante destacar los beneficios del acceso a los REA como este libro digital gratuito en cuanto a que apunta a reducir el costo de los libros de textos necesarios, que a veces pueden representar una parte significativa de los ingresos de una familia de bajos salarios. Además, no todas las universidades tienen los medios para contar con recursos digitales con altos estándares de calidad pedagógicos y tecnológicos, y por lo tanto resulta sumamente impactante y provechoso para las instituciones de países en vías de desarrollo.

\section{MATERIAL Y MÉTODO}

\section{Diseño y elaboración del libro digital Anatomía de las arterias de la cabeza}

Se generó una propuesta de construcción colaborativa entre docentes de anatomía humana de la Universidad Austral de Chile y la Universidad Autónoma de Aguascalientes de México, que fue aprobada para construir el libro digital.

Entre los docentes se organizó la escritura en la plataforma del Proyecto LATin, se distribuyeron los capítulos del libro, se coordinó la estructura y armado de las unidades. Los capítulos fueron entregados en formato Word y posteriormente subidos a la plataforma a modo de copiar y pegar de acuerdo a las secciones ya distribuidas. 
En la composición de los capítulos, se decidió que temas que requerían cierta jerarquización y calzaban con una presentación a modo de mapas conceptuales digitales, se generaran como tales con el software Inspiration, que permite el uso de colores por niveles y ordenamiento en escala.

Se tomaron fotografías de cuello y cabeza de material cadavérico, previamente disecado para los fines del libro digital, perteneciente al Pabellón de Anatomía de la Universidad Austral de Chile (Fig 1).

El equipo del Proyecto LATin se encargó del diseño de portada, licenciamiento y maquetación final del libro digital (Fig. 2).

36

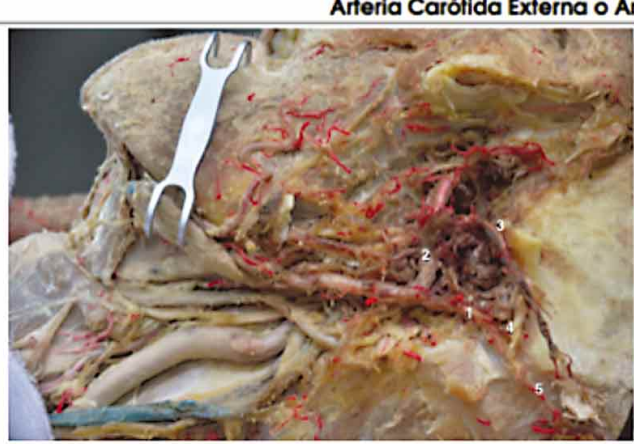

Figura 3.17: Arteria temporal superficial (1) y sus ramas arteria frontal (4) y parietal (5). Se observa la arteria maxilar (2) y la arteria temporal profunda posterior (3).

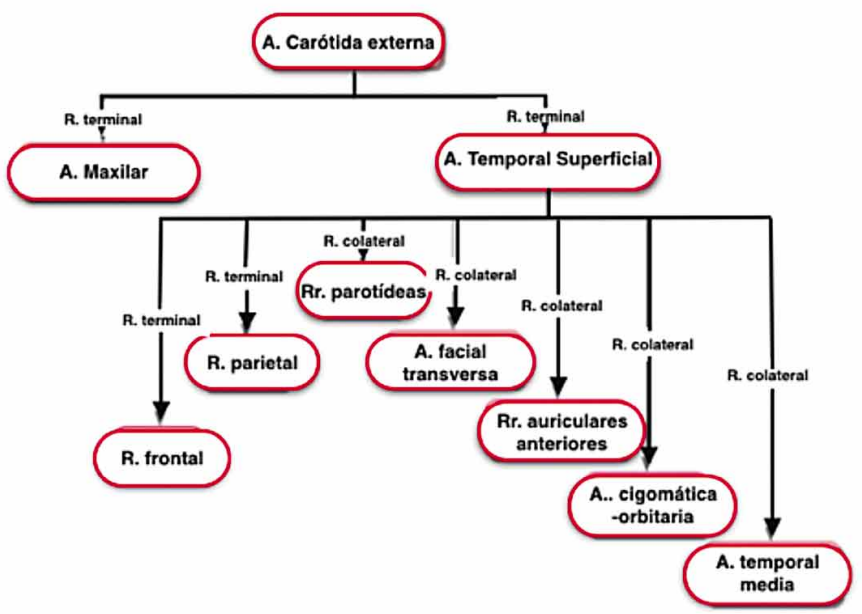

Figura 3.18: Mapa conceptual de las ramas colaterales y terminales de la a. temporal superficial que es rama terminal de la a. carótida externa.

la dirección que toman después de su emergencia: $1^{\circ}$ ramas colaterales ascendentes; $2^{\circ}$ ramas colaterales descendentes; $3^{\circ}$ ramas colaterales anteriores y; $4^{\circ}$ ramas colaterales posteriores (ver Fig. 3.19).

Fig. 1. Página del tercer capítulo del libro donde se muestra una fotografía de la arteria temporal superficial de un cadáver diseccionado en el laboratorio de anatomía humana de la Universidad Austral de Chile y posteriormente la esquematización de sus ramas terminales y colaterales.
Una vez finalizado el libro y dispuesto en Internet, este fue utilizado los primeros semestres de cada año en la asignatura Anatomía Funcional Básica dirigida a estudiantes de la carrera de Obstetricia y Puericultura. Una vez vivida la experiencia de uso didáctico de este recurso por parte de los estudiantes, durante los semestres correspondientes a los años 2014 y 2015, se aplicó una encuesta de satisfacción para tener una primera valoración acerca del REA.

\section{Diseño de la encuesta}

Con la colaboración de la profesora Ilse Pradenas de la Unidad de Anatomía de la Universidad de Antofagasta, se elaboró y aplicó a dichos estudiantes una encuesta de satisfacción del recurso, la que permitió evaluar los aspectos técnicos-estéticos y pedagógicos del programa. 


\begin{abstract}
El objetivo es conocer su opinión sobre el libro digital Anatomía de las Arterias de la Cabeza y cómo este puede ser un aporte a su aprendizaje. Además, de este modo, la evaluación nos permitirá detectar problemas, si los hubiere, $y$ mejorar el recurso tanto en sus aspectos téenicos como pedagógicos.
\end{abstract}

Por favor conteste sinceramente:

1- Aspectos técnicos.

Presenta originalidad y uso de tecnologías avanzadas: Si:

No:

Es fácil abrir el recurso: Si:

No:

Calidad y relevancia de imágenes: Si:

No:

Calidad y relevancia del texto: Si:

No:

Combina adecuadamente Imagen -texto: Si:

No:

2- Marque con una $X$ la celda que mejor interprete la afirmación:

\begin{tabular}{|l|l|l|l|l|}
\hline \multicolumn{1}{|c|}{ AFIRMACIONES } & $\begin{array}{c}\text { Muy } \\
\text { adecuado/a }\end{array}$ & $\begin{array}{c}\text { Bastante } \\
\text { adecuado/a }\end{array}$ & Adecuado/a & $\begin{array}{c}\text { Poco } \\
\text { adecuado/a }\end{array}$ \\
\hline $\begin{array}{l}\text { El recurso motiva el } \\
\text { aprendizaje }\end{array}$ & & & \\
\hline $\begin{array}{l}\text { El contenido pedagógico es } \\
\text { relevante. }\end{array}$ & & & \\
\hline $\begin{array}{l}\text { Nivel de claridad de la } \\
\text { información presentada. }\end{array}$ & & & \\
\hline $\begin{array}{l}\text { La redacción del texto resulta } \\
\text { clara para Ud. }\end{array}$ & & & \\
\hline $\begin{array}{l}\text { Fomenta el auto-aprendizaje } \\
\text { (iniciativa, toma de } \\
\text { decisiones) }\end{array}$ & & & & \\
\hline $\begin{array}{l}\text { Posibilidades de adaptación } \\
\text { a diferentes usuarios. }\end{array}$ & & & & \\
\hline $\begin{array}{l}\text { Posibilita el trabajo } \\
\text { colaborativo. }\end{array}$ & & & & \\
\hline
\end{tabular}

Comentario u opinión que considere importante para mejorar el recurso:

Fig. 3. Imagen de la encuesta elaborada para evaluar la satisfacción del libro Anatomía de las Arterias de la Cabeza.

Para registrar aspectos técnicos, se utilizó una variante dicotómica (sí-no) para registrar el grado de acuerdo. En los aspectos pedagógicos, se realizaron 7 afirmaciones con 4 alternativas de respuesta en una escala ordinal tipo Lickert (poco de acuerdo a muy de acuerdo). Para efectos de estadística descriptiva, se describe el grado de acuerdo/ no acuerdo mediante escala dicotómica (Fig. 3).

Los datos sociodemográficos de los participantes y los resultados de la escala se exponen mediante estadística descriptiva analizados con el software STATA versión 10.0.

\section{RESULTADOS}

El año 2014 se aplicó la encuesta a 51 estudiantes con un promedio de edad de 19,1 años en el cual el $91 \%$ correspondía a mujeres, en tanto que el año 2015 se encuestaron 50 estudiantes con un promedio de edad de 19 años, donde el $88 \%$ correspondía a mujeres. La encuesta consistió en evaluar los aspectos técnicos y pedagógicos del libro. Los resultados obtenidos se describen en las Tablas I y II. 
Tabla I. Resultados de evaluación de aspectos técnicos.

\begin{tabular}{lcc}
\hline Aspectos Técnicos & $\begin{array}{c}\text { 2014 Afirmaciones } \\
\text { (Sí)\% }\end{array}$ & $\begin{array}{c}\text { 2015 Afirmaciones } \\
\text { (Sí)\% }\end{array}$ \\
\hline Presenta originalidad y uso de tecnologías avanzadas & 92,2 & 80 \\
Es fácil abrir el recurso & 100 & 98 \\
Calidad y relevancia de imágenes & 78,4 & 86 \\
Combina adecuadamente imagen-texto & 88,2 & 100 \\
\hline
\end{tabular}

Tabla II. Resultados de evaluación de aspectos pedagógicos.

\begin{tabular}{lcc}
\hline Aspectos Pedagógicos & $\begin{array}{c}\text { Año 2014. } \\
\text { Afirmaciones\% }\end{array}$ & $\begin{array}{c}\text { Año 2015. } \\
\text { Afirmaciones \% }\end{array}$ \\
\hline El recurso motiva el aprendizaje & 78,5 & 100 \\
El contenido pedagógico es relevante & 94,1 & 100 \\
Nivel de claridad de la información presentada & 78,4 & 100 \\
La redacción del texto resulta clara para usted & 84,3 & 100 \\
Fomenta el auto-aprendizaje (iniciativa, toma de decisiones) & 76,4 & 98 \\
Posibilidades de adaptac ión a diferentes usuarios & 76,4 & 98 \\
Posibilita el trabajo colaborativo & 84,3 & 96 \\
\hline
\end{tabular}

\section{DISCUSIÓN}

Hay consenso, y es por lo demás con toda razón, que no se puede volver a contar con la disponibilidad de tiempo que se otorgaba a la enseñanza de la anatomía. Los tiempos han cambiado conjuntamente con la emergencia de tecnologías y nuevas metodologías de enseñanza-aprendizaje centradas en el estudiante. Por lo demás, si pensamos en la enseñanza de la anatomía humana como la de otras disciplinas básicas, hay contenidos que por su aplicación a la clínica, adquieren una gran relevancia y cuyo aprendizaje debe ser comprendido y retenido en forma eficiente (Guiraldes et al., 2001). Frente a este contexto, y al convencimiento de la relevancia del uso del material cadavérico en la enseñanza de la anatomía humana, hay que mejorar los métodos y la calidad, fortaleciendo fuertemente las actividades prácticas con el apoyo de las tecnologías, previo y durante estas actividades. En el caso de la Universidad Austral de Chile, por las características actuales de aumento de estudiantes y poco material orgánico disponible para la enseñanza, se hace indispensable buscar el apoyo eficiente de las tecnologías de aprendizaje y abordar, por lo demás en un mundo globalizado, la democratización de la enseñanza, disponiendo de recursos educativos de altos estándares para cualquier estudiante de habla hispana en cualquier parte del mundo.

El libro Anatomía de las Arterias de la Cabeza, como un primer esfuerzo por disponer de un REA, usado por estudiantes de Obstetricia en la asignatura de 1er año de anatomía para las promociones 2014 y 2015, demostró altos niveles de adaptación y uso, por parte de estos usuarios tanto en sus componentes técnicos como pedagógicos.
En los aspectos técnicos, los aspectos con más baja validación por parte de los estudiantes fue en el 2014, por calidad y la relevancia de imágenes $(78,4 \%)$. En tanto que este aspecto fue el mejor evaluado por la promoción 2015 (86\%), quienes, sin embargo, mostraron una apreciación más baja $(80 \%)$ respecto del ítem, presenta originalidad y uso de tecnologías avanzadas. Será conveniente revisar si las imágenes están bien posicionadas dentro del texto y responden bien a lo que se desea mostrar.

En cuanto a los aspectos pedagógicos evaluados, coincidentemente, por ambas promociones de alumnos, los ítems: fomenta el auto-aprendizaje y posibilidades de adaptación a diferentes usuarios, fueron los de menor apreciación: los dos con una valoración de 76,4 \% y $98 \%$ respectivamente en ambos años. En contraste, la más alta valoración se otorgaron a los ítems: el contenido pedagógico es relevante con 94,1\% el 2014 y la redacción del texto resulta clara para usted alcanzó $84,3 \%$ el 2014, en cambio, la promoción del 2015 consideró esto y los demás ítems con un 100 de afirmación. Se aprecia un leve grado de dificultad para aceptar este recurso educativo como de auto-aprendizaje y de uso para diferentes usuarios en la promoción de 2014, por lo que se propone nuevo análisis en cuanto a estos parámetros y realizar estas mediciones en otras carreras del área de la salud, así como con alumnos de otras universidades.

La eficiencia se puede alcanzar haciendo uso de recursos multimediales, que integrados de una manera ade- 
cuada, pueden hasta incluso reducir el tiempo de comprensión de contenidos complejos en libros tradicionales.

Lo mencionado en el párrafo anterior ocurre con este tipo de REA -el libro digital- se considera un REA por la definición de este último como recurso educativo de licencia abierta, posibilitada por las tecnologías de la información y la comunicación para la consulta, uso y adaptación por parte de una comunidad de usuarios sin fines comerciales (Inamorato et al.). Por sus características, en que la integración de sus contenidos es muy novedosa en esta área del conocimiento, esto porque integra diversos recursos multimediales en forma cuidadosa, como el texto necesario y suficiente; en este caso se incorporaron mapas conceptuales digitales, fotografías reales, además de los textos correspondientes.
Finalmente, gracias a la evaluación y uso de este recurso por parte de estudiantes, es que se planea materializar el año 2017, una segunda edición de este libro digital.

\section{AGRADECIMIENTOS}

Muchas gracias a los estudiantes de la Escuela de Obstetricia y Puericultura de las promociones 2014 y 2015 que contestaron las encuestas con la mejor de las dispociones. Se agradece también al equipo del Proyecto LATin, como al Proyecto MECESUP AUS 1410 por hacer posible la fifusión de REA.

BUCAREY, A. S.; ARAVENA, T. P.; PRADENAS, M. I.; TIZNADO, M. G. Anatomy of the arteries of the head: An open educational resource as a digital tool to support the teaching of anatomy. Int. J. Morphol., 34(4):1285-1292, 2016.

SUMMARY: According to anatomists and students around the world, the study of this discipline requires practical work in front of the body. In light of the reduction of time in the teaching of this discipline, these stages must be strongly supported by educational technologies and in turn meet the demands of a globalized society that promotes equity of Education. In this context, the "Open Educational Resources" (OER) is a great tool. The OER movement has had significant success in promoting the idea that knowledge is a universal asset. Since its inception, the OER were consolidated as key to the democratization of knowledge, because they are available online with licensed Creative Commons. Through the Latin American Initiative open textbooks from Latin Project, a digital book for students of health called "Anatomy Arteries of the Head" was organized in such a way to properly integrate content, as multimedia from an pedagogical perspective. The book meets the characteristics of a OER, since it is available and easily accessible on the Internet, also through Latin Project is properly distributed in the 12 universities that make up the project network. This book is used in the Circulatory System unit, in the course of Human Anatomy (ANAT 122) that was issued to students at the Midwifery School the first half of 2014 and first half 2015. The book was evaluated by a total of 101 students with a descriptive study to determine satisfaction from the pedagogical and technological perspective. The results showed a very high satisfaction with the resource, fluctuating between $76,4 \%$ and $100 \%$ satisfaction in various items.

KEY WORDS: Anatomy; Teaching; Open Educational Resources.

\section{REFERENCIAS BIBLIOGRÁFICAS}

Arboleda Toro, N. \& Rama Vitale, C. La Educación Superior a Distancia y Virtual en Colombia: Nuevas Realidades. Bogotá, Virtual Educa y Asociación Colombiana de Instituciones de Educación Superior con Programas a Distancia y Virtual, 2013.

Bravo, H. Plastination an additional tool to teach anatomy. Int. J. Morphol., 24(3):475-80, 2006.

Bergman, E. M.; Prince, K. J.; Drukker, J.; van der Vleuten, C. P. $\&$ Scherpbier, A. J. How much anatomy is enough? Anat. Sci. Educ., 1(4):184-8, 2008.

Bucarey, S.; Cárdenas, F.; Esperón, R. \& Aguilar, M. Educación a Distancia: Una mirada a experiencias de universidades chilenas y latinoamericanas. Valdivia, Universidad Austral de Chile, 2013.
Ehlers, U. D. Extending the territory: From open educational resources to open educational practices. J. Open Flex. Distance Learn., 15(2):1-10, 2011.

Fitzgerald, J. E.; White, M. J.; Tang, S. W.; Maxwell-Armstrong, C. A. \& James, D. K. Are we teaching sufficient anatomy at medical school? The opinions of newly qualified doctors. Clin. Anat., 21(7):718-24, 2008.

Guiraldes, H.; Oddó, H.; Mena, B.; Velasco, N. \& Paulos, J. Teaching human anatomy: Experience and challenge in a medical school. Rev. Chil. Anat., 19(2):205-12, 2001.

Inamorato, A.; Cobo, C. \& Costa, C. Compendio Recursos Educativos Abiertos: Casos de América Latina y Europa en la Educación Superior. Niterói, Universidad Federal Fluminense, 2012. Disponible en: http://www.oportunidadproject.eu 
BUCAREY, A. S.; ARAVENA, T. P.; PRADENAS, M. I.; TIZNADO, M. G. Anatomía de las arterias de la cabeza: un recurso educativo abierto como herramienta digital de apoyo a la enseñanza de la anatomía. Int. J. Morphol., 34(4):1285-1292, 2016.

Inzunza, O. Morphology, the new challenges for 2015. Int. J. Morphol., 32(3):789-93, 2014.

Inzunza, O. \& Bravo, H. Computer animation of photographs, an actual contribution to practical learning of human anatomy. Rev. Chil. Anat., 20(2):151-7, 2002.

Kurkcuoglu, A.; Pelin, C.; Zagyapan, R. \& Ogus, E. Opinions of medical students about phase i anatomy education: a preliminary study. Rev. Arg. Anat. Clin., 7(1):26-33, 2015.

Nayak, S.; Ramnarayan, K.; Somayaji, N. \& Bairy, K. L. Teaching anatomy in a problem-based learning (PBL) curriculum. Neuroanatomy, 5:2-3, 2006.

Patel, K. M. \& Moxham, B. J. Attitudes of professional anatomists to curricular change. Clin. Anat., 19(2):132-41, 2006.

Ramírez, M. S. Administración de Objetos de Aprendizaje en Educación a Distancia: Experiencia de Colaboración Interinstitucional. En: Lozano, A. \& Burgos, V. (Eds.). Tecnología Educativa en un Modelo de Educación a Distancia Centrado en la Persona. Ciudad de México, Limusa. 2007. pp.356-7.

Universidad Virtual-REUNA. La Educación Virtual en Chile: Historia, Estado del Arte y Proyecciones hacia el Conocimiento de la Realidad Nacional en Materia de Uso de Tecnología Aplicada a la Educación Superior. Santiago de Chile, 2003. Disponible en: http://unesdoc.unesco.org/images/0014/ 001403/140393s.pdf

Waterston, S. W. \& Steward, I. J. Survey of clinicians' attitudes to the anatomical teaching and knowledge of medical students. Clin. Anat., 18(5):380-4, 2005.

\author{
Dirección para correspondencia: \\ Sandra Bucarey Arriagada \\ Instituto de Anatomía, Histología y Patología \\ Facultad de Medicina \\ Edificio de Ciencias Biomédica \\ Campus Isla Teja \\ Valdivia \\ CHILE
}

Email: sbucarey@gmail.com

Recibido : 28-03-2016

Aceptado: 11-08-2016 